\title{
New insights into the anti-inflammatory actions of aspirin- induction of nitric oxide through the generation of epi-lipoxins Derek W Gilroy
}

\author{
Centre for Clinical Pharmacology and Therapeutics, BHF Laboratories, Division of Medicine, University College London, \\ 5 University Street, London WC1E 6JJ, UK
}

\begin{abstract}
Aspirin has always remained an enigmatic drug. Not only does it present with new benefits for treating an everexpanding list of apparently unrelated diseases at an astounding rate but also because aspirin enhances our understanding of the nature of these diseases processe. Originally, the beneficial effects of aspirin were shown to stem from its inhibition of cyclooxygenase-derived prostaglandins, fatty acid metabolites that modulate host defense. However, in addition to inhibiting cyclooxygenase activity aspirin can also inhibit pro-inflammatory signaling pathways, gene expression and other factors distinct from eicosanoid biosynthesis that drive inflammation as well as enhance the synthesis of endogenous protective anti-inflammatory factors. Its true mechanism of action in anti-inflammation remains unclear. Here the data from a series of recent experiments proposing that one of aspirin's predominant roles in inflammation is the induction of nitric oxide, which potently inhibits leukocytelendothelium interaction during acute inflammation, will be discussed. It will be argued that this nitric oxide-inducing effects are exclusive to aspirin due to its unique ability, among the family of traditional anti-inflammatory drugs, to acetylate the active site of inducible cyclooxygenase and generate a family of lipid mediators called the epi-lipoxins that are increasingly being shown to have profound roles in a range of host defense responses.
\end{abstract}

Key words: eicosanoids - cell trafficking - acute inflammation

After carrying our what might be considered the world's first clinical trial on salicylate the Reverend Edward (Edmund) Stone presented his findings to the Royal Society in 1763 writing that I have no other motives for publishing this valuable specific, than it may have fair and full trial in all its variety of circumstances and situations, an that the world may reap the benefits accruing from it. In his trial he administered a crude extract of Willow bark to 50 willing volunteers experiencing fever with apparent safety and success (Vane et al. 1992). Could Stone have ever imagined the impact of his prediction on the use of aspirin for the treatment of inflammation and fever? Of course salicylate is not used today, it was found to be too unpalatable. In an attempt to find a more compliant but equally efficacious form, salicylate was acetylated by Felix Hoffmann in 1897 and thus aspirin was born. The scope of this review does not allow for the complete testimonial that aspirin's colorful history deserves; from the usage of salicylate in antiquity for inflammation and pain to the countless indications of acetylated salicylate today including cardiovascular diseases, stroke, pregnancy complications, cancer, diabetes, Alzheimer's disease etc. But, just like the efforts made throughout the 1800's to elucidate the structure of salicylate and find an alternative which culminated in aspirin, huge efforts were being made through the 1900's to understand how aspirin works. In this short review, some of the earlier theories on aspirin's actions will be recounted including Vane's ground-break-

E-mail: d.gilroy@ucl.ac.uk

Received 8 November 2004

Accepted 30 December 2004 ing discovery of prostaglandin (PG) inhibition. Equally, some of the questions surrounding the PG inhibition theory will be discussed as will the alternatives that have made the literature in recent years culminating in what we believe is how aspirin works, in animals models of acute inflammation at least - the induction of nitric oxide (NO).

\section{How aspirin works - The traditional view}

By the early 1970's comparatively little was known about the soluble mediators that drive the inflammatory response let alone how aspirin and the other aspirin-like drugs might be altering them. It was suggested, for example, that aspirin-like drugs uncoupled oxidative phosphorylation (Whitehouse et al. 1962), inhibited dehydrogenase enzymes dependent upon pyridine nucleotides (Hines et al. 1964) as well as inhibited some amino transferases and decarboxylases (Gould et al. 1965). The major inconsistency with these theories was that the levels of aspirin required to inhibit these enzymes were much higher than the plasma level achieved by anti-inflammatory doses of aspirin and that inhibition of some or even all of these enzymic pathways could not explain aspirins anti-inflammatory, anti-pyretic, and analgesic properties. One of the most promising ideas at the time was that aspirin and the salicylates could inhibit proteases found at inflammatory sites and which were found to cause tissue damage associated with chronic inflammatory disease processes. Notwithstanding, a convincing and consistent mechanism was lacking.

Perhaps the first step in the aspirin/PG story came from the pioneering work of Collier and colleagues, who crucially identified that aspirin inhibited the bronchoconstriction elicited by bradykinin (Collier et al. 1960) and the slow-reacting-substances of anaphylaxis (Berry et al. 1964). One of his graduate students, Pricilla Piper, went to 
work with Sir John Vane at the Royal College of Surgeons to further understand how aspirin exerted its protective effect against bronchoconstriction. During the course of their studies they identified a number of novel factors that were released during anaphylaxis including $\mathrm{PGE}_{2}$, PGF $2 \alpha$ and what was called at the time rabbit aorta contracting substance, subsequently identified as thromboxane $A_{2}$ (Vane et al. 1992). This introduced the first hint of an eicosanoid link in the aspirin story. Again, irreverently skipping through vast swathes of highly pertinent history Vane and colleagues went on to show that aspirin could inhibit thromboxane and PG synthesis in a number of tissues from various species in response to a vast array of stimuli. Hindsight allows us now to see the obvious connection between aspirin and the inhibition of PGs though what was not known at the time was how PGs were made. It was clear that there was a certain level of PGs constitutively present in most tissues and Vane noticed that their levels increased in response to their various stimulating protocols and concluded that PGs synthesis could be enhanced. Vane, admitting that the wood was not then apparent from the trees, suggested that aspirin must be inhibiting the enzyme system that made PGs. And so it did. Vane made history in 1971 when he demonstrated that guinea pig lung homogenates incubated with aspirin and other non-steroidal anti-inflammatory drugs dose-dependently inhibited the ability of these tissues to make PGs. Work by Smith and Willis corroborated this concept by publishing in the same issue of Nature that aspirin and other non-steroidal anti-inflammatory agents inhibited PG synthesis from platelets, the importance of which was reinforced by the fact that not alone were these studies done on guinea pig lungs but that aspirin was effective in this regard after oral administration in man (Smith et al. 1971). Unarguably, then, the mystery was solved, or was it?

\section{Problems with the prostagladin theory}

Aspirin is unique among the established non-steroidals in that it has effects on both the inflammatory response and cardiovascular system. For instance, it protects against platelet aggregation in humans at $75 \mathrm{mg}$ whereas $1 \mathrm{~g}$ four times daily is required to treat chronic inflammatory diseases such as rheumatoid arthritis. Its protective effects against platelet aggregation are due to the inhibition of thromboxane $A_{2}$. Thus, aspirin can inhibit PG synthesis at doses that are considerably lower than that which inhibits inflammation suggesting that at the higher doses of $1 \mathrm{~g}$ aspirin must be inhibiting, inducing or otherwise altering some other pathways pertinent to the inflammatory response in humans other than arachidonic acid metabolism. One argument against this is that the cardioprotective dose of aspirin does not inhibit all tissue PGs, which may be sufficient to maintain the inflammatory response. Another, more convincing argument pertaining to the aspirin/PG theory lies in the fundamental properties of the PGs. Classically, cyclooxygenase metabolises arachidonic acid to the PGs including $\mathrm{PGE}_{2}$, $\mathrm{PGI}_{2}, \mathrm{PGD}_{2}$ and $\mathrm{PGI}_{2}$, which together with thromboxane $\mathrm{A}_{2}$ constitute the prostanoids. In general the PGs, acting in synergy with other mediators such as histamine and bradykinin, play a role in oedema formation and contribute to pain but are not generally considered to be chemoattractant for leukocytes (Williams et al. 1988). Within the eicosanoid family, this is the responsibility of the leukotrienes, in particular leukotriene $\mathrm{B}_{4}$, a metabolite of the 5 lipoxygenase (LOX) pathway of arachidonic metabolism. Therefore, how can the anti-leukocyte accumulating properties of aspirin be explained by inhibiting PGs if such fatty acid hormones play little role in cell trafficking? Many elegant experiments have been performed to help address this short fall in our understanding of how aspirin works. For instance, aspirin has been shown to inhibit NF-kB activation (Kopp et al. 1994), mediate the release of adenosine (Cronstein et al. 1999), activate heat shock proteins (Fawcett et al. 1997) and down-regulate inducible COX expression (Wu et al. 1991), to name but a few. However, these bio-effects of aspirin are also shared with salicylate, indicating that many of the anti-inflammatory properties of aspirin may, in fact, be due to salicylate. But even the reported bio-effects of salicylate do not correlate with anti-inflammation. For instance, salicylate was shown to inhibit COX 2 mRNA expression in LPS-elicited alveolar macrophages at $30 \mathrm{mg} / \mathrm{kg}$ (Wu et al. 1991), dosing levels that are far below that which are anti-inflammatory in mice, leading us to speculate that under some circumstances the diverse effects of salicylate maybe secondary to that of aspirin. However, perhaps one of the most exciting aspects of the mechanism of action of aspirin stems from its ability to trigger lipoxin synthesis (so-called aspirin-triggered epi-lipoxins) as a result of acetylating the active site of COX 2 in endothelial or epithelial cells, a property not shared with other NSAIDs (Claria et al. 1995). This results in the synthesis of $(15 R)$ hydroxyeicosatetraenoic acid (15R-HETE), which is rapidly metabolised in a transcellular manner by leukocyte 5-lipoxygenase to potently anti-inflammatory 15 -epi-lipoxin $\mathrm{A}_{4}$ or $\mathrm{B}_{4}$. Is it possible that the anti-inflammatory effects of aspirin are due not to the inhibition of pro-inflammatory signals but to lipoxin biosynthesis? Certainly, attempting to solve the enigma of aspirin's anti-inflammatory actions would consume a vast amount of time and money. But perhaps the best strategy for those of us enthused enough to pursue this issue is to include aspirin in any treatment group in your experiments when you are looking at a factor or parameter never before examined. This is what we did when carrying out some early experiments looking at the role of NO in acute inflammation.

\section{Aspirin and NO}

An extra group of rats bearing an acute pleuritis were given a single anti-inflammatory dose of aspirin, which caused high levels of nitrite/nitrate to appear in the peripheral blood of these animals after a few hours. On repeat, we found a reduction in local inflammation that correlated inversely with a dose-dependent and significant increase in plasma NO levels as measured by nitrite using the Griess reaction (Fig. 1). This was in contrast to other nonsteroidal anti-inflammatory drugs such as salicylate, piroxicam, and indomethacin which had no effect on plasma nitrite. This observation was exciting for two reasons. First, NO has profound protective effects on the cardio- 
vascular system and second, it inhibits leukocyte trafficking. It is NO's ability to prevent leukocyte/endothelial cell interaction during acute inflammation that interested us most. After a series of experiments we found that inhibiting aspirin-elicited NO pharmacologically nullified the anti-inflammatory effects of aspirin. Moreover, aspirin was not anti-inflammatory in either constitutive (eNOS) or inducible NO synthase (iNOS) knockout mice with IL-1 $\beta$ induced peritonitis. It transpired that aspirin generated NO through its unique ability to trigger the synthesis of 15-epi-lipoxin $\mathrm{A}_{4}$. Aspirin and 15-epi-lipoxin $\mathrm{A}_{4}$ were shown to inhibit leukocyte trafficking in an NO-dependent manner using intravital microscopy on IL-1 $\beta$-stimulated mouse mesentery. Not only did aspirin inhibit leukocyte-endothelial interaction in a manner similar to $\mathrm{NO}$ in wild-type mice but both aspirin and 15-epi-lipoxin $\mathrm{A}_{4}$ had markedly reduced effects on leukocyte-endothelial cell adherence in eNOS- and iNOS-deficient mice compared with wild type (Paul-Clark et al. 2004). From these findings we concluded that once ingested, aspirin is exposed to the vasculature where it acetylates COX 2 within the endothelium or circulating leukocytes to trigger 15-epilipoxin $\mathrm{A}_{4}$ which, in turn, elicits NO synthesis from both eNOS and iNOS. Ultimately, aspirin-triggered NO mediates the anti-inflammatory effects of aspirin in the microcirculation by negatively regulating leukocyte/en-dothelium interaction (Fig. 2A, B).

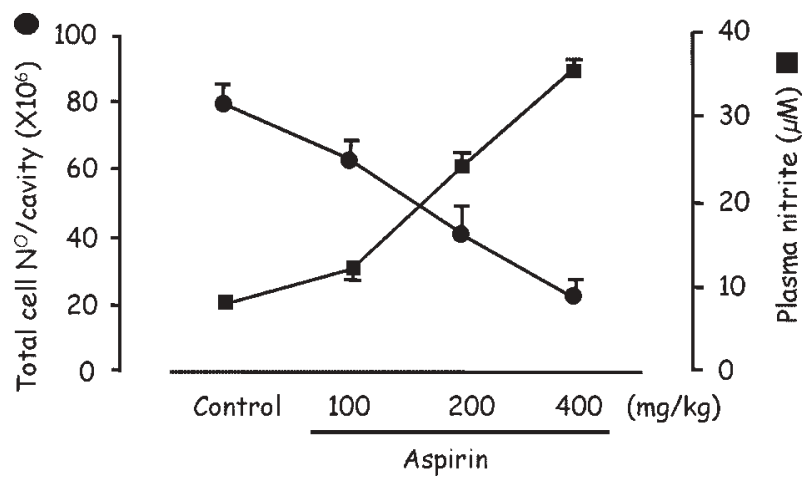

Fig. 1: the effects of aspirin on nitric oxide-synthesis in the peripheral blood of rats bearing a carrageenan-induced pleurisy. Aspirin was dosed orally $1 \mathrm{~h}$ before the intrapleural injection of carrageenan and inflammation and peripheral blood sampled $4 \mathrm{~h}$ after stimulus.

\section{What have these studies told us about the innate in- flammatory response?}

Endogenous NOS regulation - In addition to showing how aspirin works in murine peritonitis, these studies have revealed quite a great deal of additional information about the onset phase of acute inflammation. Firstly, it tells us that in addition to aspirin, the epi-lipoxins also regulate cell trafficking through NO. Secondly, it seems that both eNOS and iNOS are required for the control of leukocyte trafficking during acute inflammation and that eNOS is required to signal subsequent iNOS expression. In terms of the regulation of NO synthesis during acute inflammation how do aspirin and the lipoxins increase NO synthesis and what cross-talk exists between eNOS and iNOS for the regulation of leukocyte trafficking in aspirintreated inflammation? The former is difficult to address at this stage. However, regarding the latter, it is known that $\mathrm{NO}$ has a biphasic effect on NF-kB activity in murine macrophages, being able to up- and down-regulate the expression of a number of pro-inflammatory proteins, including iNOS (Connelly et al. 2001). It appears that low levels of NO, as produced from eNOS, activate NF-kB whereas higher levels of NO, typical of iNOS, inhibit NF$\mathrm{kB}$. In support of this, it was recently shown that lipopolysaccharide-stimulated bone marrow derived macrophages from eNOS knockout mice show greatly reduced NF-kB activity and iNOS expression compared to wild type cells (Connelly et al. 2003). These authors demonstrated that eNOS triggered iNOS expression, in part, through soluble guanylate cyclase (sGC) as enhanced iNOS expression, brought about by levels of NO similar to that produced by eNOS, were inhibited by ODQ (a sGC inhibitor) but increased by BAY 41-2272 (sGC activator). As NO can activate NF-kB in a sGC-dependent manner, the potentiating effects of $\mathrm{NO}$ on NF-kB activity and subsequent iNOS expression in macrophages are likely to occur, at least in part, via activation of sGC and the production of cGMP. A critical role for this NO/cGMP pathway in cytokine signaling of iNOS expression was also shown in human mesangial cells (Pérez-Sala et al. 2001). Thus, inhibiting the first wave of NO stimulated by IL-1 $\beta$ or tumor necrosis factor with L-NAME resulted in a subsequent reduction in iNOS expression and activity, which was reversed using an NO donor. Induction of iNOS was shown to be sGC-dependent as ODQ reduced cytokine and NO donor-stimulated iNOS expression, effects that were rescued with a cell-permeable cGMP analogue. Collectively, these studies suggest that if the initial NO-mediated response to stimulation is absent, the magnitude of subsequent iNOS expression is diminished. These in vitro findings may explain why plasma $\mathrm{NO}$ levels in aspirin-treated eNOS $^{-/-}$and iNOS $^{-/-}$mice are much lower than aspirintreated wild type animals. In the absence of eNOS, for instance, iNOS expression maybe reduced producing correspondingly low amounts of NO. Similarly, in the absence of iNOS, though eNOS activity maybe enhanced slightly by aspirin, levels of NO from this constitutive isoform will also be low. While there is a possible contribution of NO from nNOS in this setting, based on the evidence so far it appears that in acute inflammation NO mediates the antiinflammatory effects of aspirin and lipoxins and that this NO comes from the initial activation of eNOS, which subsequently triggers iNOS expression. Thus, activation of eNOS may be central to the anti-inflammatory actions of aspirin and the lipoxins.

Further insights into COX 2 in acute inflammation and the cardiovascular system - In addition to animals bearing a localized inflammation, we found that aspirin and 15-epi-lipoxin $\mathrm{A}_{4}$ also elicited $\mathrm{NO}$ synthesis in naïve animals, i.e. mice without any systemic or localized inflammatory response (Paul-Clark et al. 2004). This suggests that the biochemical machinery required to manufacture NO in response to aspirin is constitutively expressed. That 
15-epi-lipoxin $\mathrm{A}_{4}$ mediates aspirin-generated NO identifies COX 2/5-lipoxygenase as this constitutively-expressed pathway. COX 2 is not classically thought of as a constitutively expressed enzyme. However, given the size of the vasculature it has been proposed that the vascular endothelium may contain focal regions or "hot spots" under stress that express COX 2 and can generate substantial amounts of COX 2-derived epi-lipoxins with aspirin treatment (Serhan et al. 2001). Certainly, it was found that a physiological level of steady laminar sheer stress, an in vitro model that mimics the average wall shear en- countered in the vasculature, caused a sustained expression of endothelial COX 2 (Topper et al. 1996). This constitutive expression of COX 2 helps to explain why we found increased plasma NO in naïve, un-inflamed animals. In addition to the endothelium, COX 2 could also be constitutively expressed in circulating leukocytes. Indeed, we found that about $10 \%$ of circulating PMNs from naïve rats constitutively express COX 2 (Tomlinson et al. 1994). However, whether it be in the endothelium or circulation leukocytes, the exact cellular source of constitutively-expressed COX 2 remains to be identified.

A
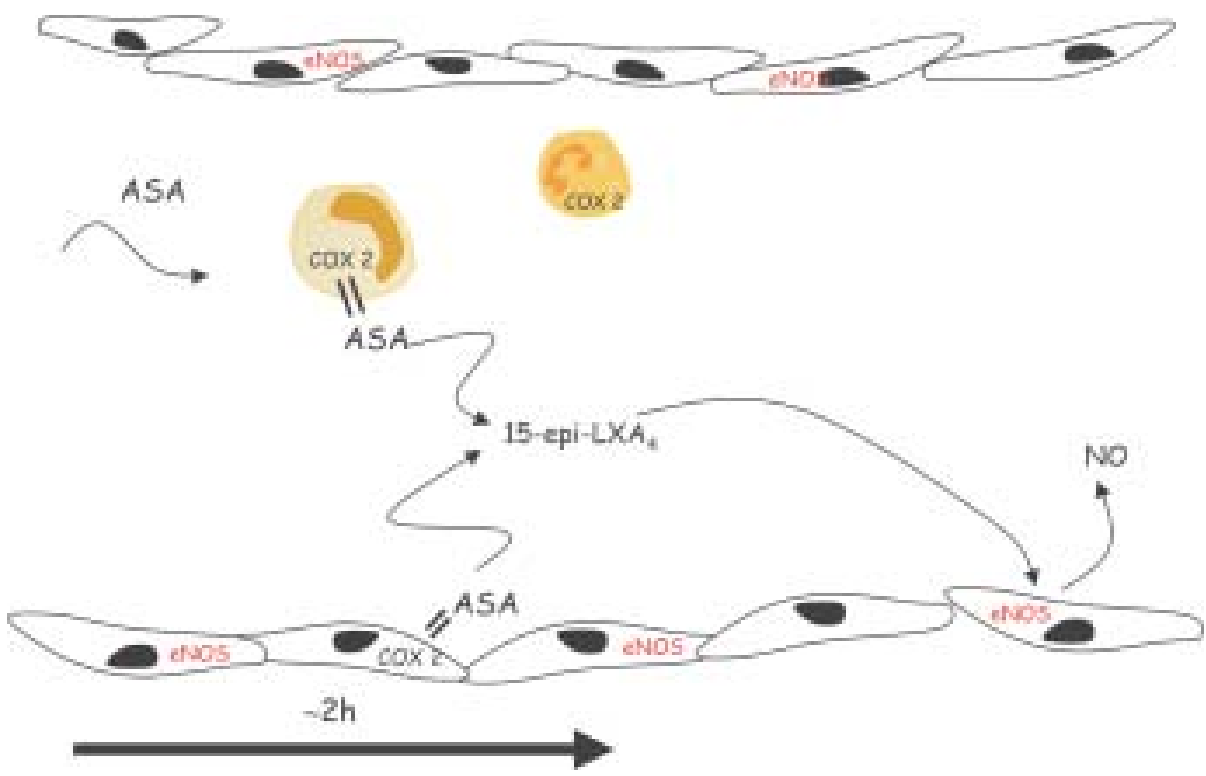

B

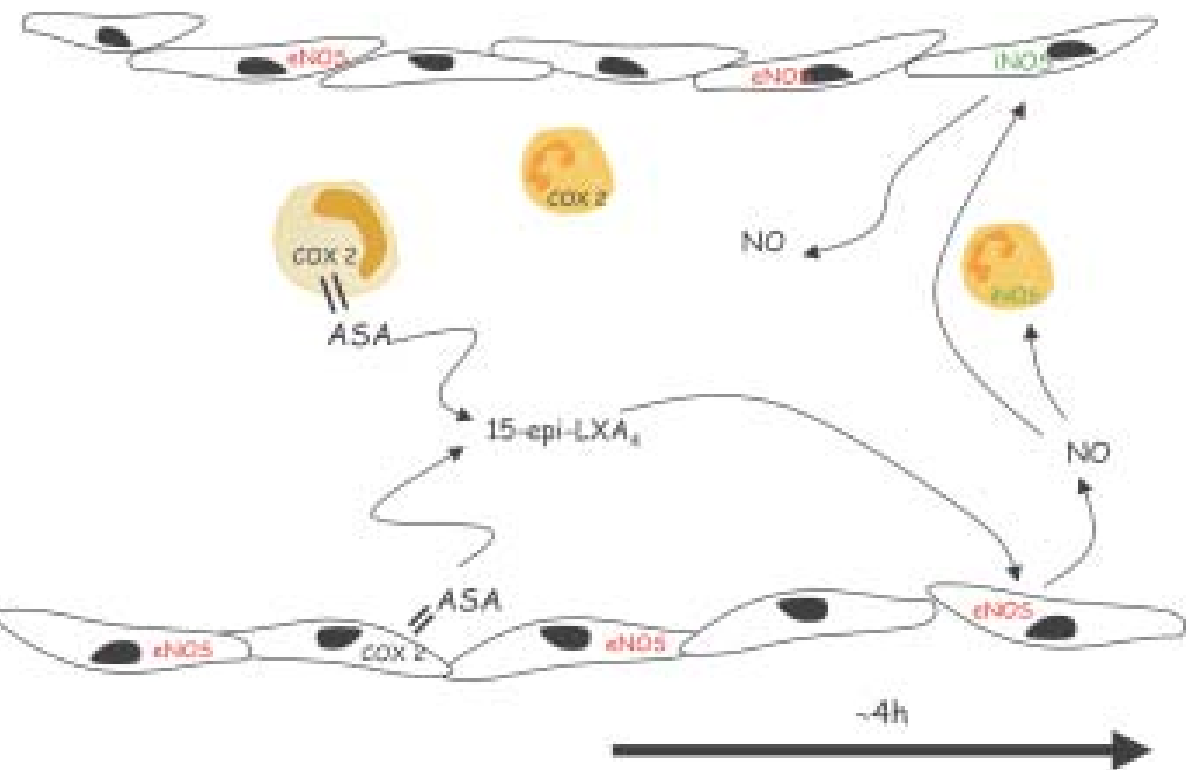

Fig. 2: hypothesis of the events occurring in the microvasculature after ingestion of aspirin. A: once in the systemic circulation aspirin acetylates constitutively-expressed COX 2 in the microvascular endothelium and/or the circulating leukocytes to generate 15-epi-lipoxin $\mathrm{A}_{4}$. This 15 epi-lipoxin $\mathrm{A}_{4}$ then triggers endothelial nitric oxide synthase (eNOS)-derived nitric oxide (NO) synthesis in an unknown manner. This eNOS-derived NO plays two roles - the control of early phase leukocyte/endothelial cell interaction (up to 2-3 h); B: the subsequent induction of inducible nitric oxide sysnthase (iNOS) in the endothelium, the leukocytes or both. NO from iNOS is then responsible for the later phase of control of leukocyte trafficking. 


\section{Contraindicating aspirin with $\mathrm{COX} 2$ inhibitors}

In addition to the established role of $\mathrm{NO}$ in negatively regulating leukocyte/endothelium interactions, the lipoxins and aspirin-triggered epi-lipoxins also regulate the extravascular accumulation of leukocytes during acute inflammation by altering leukocyte/endothelial interaction (Filep et al. 2002) as well as having a direct effect on leukocyte diapedesis (Perretti et al. 2002) and chemotaxis (Colgan et al. 1993). For instance, lipoxin $\mathrm{A}_{4}$ stable analogues modulate the expression of both L-selectin and CD11/CD18 on resting and stimulated leukocytes and inhibit neutrophil adhesion to human coronary artery endothelial cells by reducing CD11/CD18 expression (Filep et al. 1999). Whether lipoxins mediate these effects on cell adhesion molecule expression directly or indirectly through $\mathrm{NO}$ is unclear. Certainly, NO is known to regulate $\mathrm{P}$-selectin (Gauthier et al. 1994) and CD11/CD18 expression (Kubes et al. 1991). Also, inhibiting aspirin-triggered 15-epi-lipoxin $\mathrm{A}_{4}$ synthesis using a selective COX 2 inhibitor or blocking the effects of 15-epi-lipoxin $\mathrm{A}_{4}$ with a lipoxin receptor antagonist markedly attenuated aspirin's inhibition of PMN/endothelial cell interaction. In these reports, this strategy of COX 2 inhibition and lipoxin receptor antagonism had only a minimal effect on anti-adhesion by an NO-releasing derivative of aspirin, NCX-4016 (Fiorucci et al. 2003, 2004). Further evidence that aspirin's protective properties may be mediated through an epi-lipoxin/NO pathway is apparent from a recent report examining the effects of NCX-4016 on clinical (Fiorucci et al. 2003) and experimental models of mucosal damage (Fiorucci et al. 2004). Volunteers who received a selective COX 2 inhibitor (celecoxib) in combination with aspirin developed twice the mucosal damage compared to aspirin alone. This was not observed when celecoxib was administered with NOreleasing NCX-4016 (Fiorucci et al. 2003). Similarly, in rats, celecoxib exacerbated aspirin-induced gastric damage and inflammation, but in combination with NCX-4016 was without effect (Wallace et al. 2004). These studies provide evidence that NO may mediate, to a large extent, the antiinflammatory effects of aspirin and aspirin-generated epilipoxins. In addition, the data presented here suggest that drugs that inhibit COX 2 may nullify the anti-inflammatory effects of aspirin, which supports the cautionary note sounded by others for patients taking aspirin in combination with COX 2 inhibitors (Fiorucci et al. 2003, Wallace et al. 2004).

\section{Concluding remarks}

The discovery by Vane in 1971 that aspirin exerts its anti-inflammatory effect by inhibiting PG synthesis has helped to explain many but not all of the properties of this enigmatic drug. Indeed, the inhibition of PGs is insufficient in itself to adequately explain how aspirin controls inflammation. In an attempt to identify its primary mechanism of action, we show that the induction of NO seems to be exclusive to aspirin and propose that once ingested, aspirin is exposed to the vasculature where it acetylates COX 2 within the endothelium or circulating leukocytes to trigger 15-epi-lipoxin $\mathrm{A}_{4}$ which, in turn, elicits $\mathrm{NO}$ synthesis from both eNOS and iNOS. Ultimately, aspirin-trig- gered NO mediates the anti-inflammatory effects of aspirin in the micro-circulation by negatively regulating leukocyte/endothelium interaction. As aspirin is without antiinflammatory effect in NO deficient animals, we believe this is the primary mode of action of aspirin and that the leukocyte/endothelial interface is its primary site of action. We believe these findings explain how aspirin inhibits acute inflammation. In addition, these data support the development of drugs that controllably elevate plasma NO to levels that inhibit leukocyte attachment to the endothelium but do not overload the system with potentially cytotoxic NO. Given the side effects of aspirin itself, perhaps the usage of NO-inducing agents such as lipoxins or their stable analogues maybe a further refinement on this theme.

\section{REFERENCES}

Berry PA, Collier HO 1964. Bronchoconstriction action and antagonism of a slow reacting substance from analphylaxis of guinea pig isolated lung. Br J Pharmacol 23: 201-216.

Claria J, Serhan CN 1995. Aspirin triggers previously undescribed bioactive eicosanoids by human endothelial cellleukocyte interactions. Proc Natl Acad Sci USA 92: 94759479.

Colgan SP, Serhan CN, Parkos CA, Delp-Archer C, Madara JL 1993. Lipoxin A4 modulates transmigration of human neutrophils across intestinal epithelial monolayers. J Clin Invest 92: 75-82.

Collier HO, Shorley PG 1960. Analgesic anti-pyretic drugs as antagonists of bradykinin. Br J Pharmacol 15: 601-610.

Connelly L, Jacobs AT, Palacios-Callender M, Moncada S, Hobbs AJ 2003. Macrophage endothelial nitric oxide synthase auto-regulates cellular activation and pro-inflammatory protein expression. J Biol Chem 9: 9.

Connelly L, Palacios-Callender M, Ameixa C, Moncada S, Hobbs AJ 2001. Biphasic regulation of NF-kB activity underlies the pro- and anti-inflammatory actions of nitric oxide. J Immunol 166: 3873-3881.

Cronstein BN, Montesinos MC, Weissmann G 1999. Sites of action for future therapy: an adenosine-dependent mechanism by which aspirin retains its antiinflammatory activity in cyclooxygenase-2 and NFkappaB knockout mice. Osteoarthritis Cartilage 7: 361-363.

Fawcett TW, Xu Q, Holbrook NJ 1997. Potentiation of heat stress-induced hsp70 expression in vivo by aspirin. Cell Stress Chaperones 2: 104-109.

Filep JG, Zouki C, Petasis NA, Hachicha M, Serhan CN 1999. Anti-inflammatory actions of lipoxin A(4) stable analogs are demonstrable in human whole blood: modulation of leukocyte adhesion molecules and inhibition of neutrophilendothelial interactions. Blood 94: 4132-4142.

Filep JG, Zouki C, Petasis NA, Hachicha M, Serhan CN 2002. Lipoxin A4 and aspirin-triggered 15-epi-lipoxin A4 modulate adhesion molecule expression on human leukocytes in whole blood and inhibit neutrophil-endothelial cell adhesion. Adv Exp Med Biol 507: 223-228.

Fiorucci S, Distrutti E, Mencarelli A, Morelli A, Laufor SA, Cirino G, Wallace JL 2003. Evidence that 5-lipoxygenase and acetylated cyclooxygenase 2-derived eicosanoids regulate leukocyte-endothelial asherence in response to aspirin. 


\section{Br J Pharmacol 139: 1351-1359.}

Fiorucci S, Distrutti E, Mencarelli A, Rizzo G, Di Lorenzo AR, Baldoni M, Del Soldato P, Morelli A, Wallace JL 2004. Cooperation between aspirin-triggered lipoxin and nitric oxide (NO) mediates anti-adhesive properties of NCX-4016 (NO-aspirin) on neutrophil-endothelial cell adherence. $J$ Pharmacol Exp Ther 309: 1174-1182.

Gauthier TW, Davenpeck KL, Lefer AM 1994. Nitric oxide attenuates leukocyte-endothelial interaction via P-selectin in splanchnic ischemia-reperfusion. Am J Physiol 267: G562G568.

Gould BJ, Smith MJH 1965. Salicylate and aminotransferases. J Pharm Pharmacol 17: 83-88.

Hines WJ, Smith MJ 1964. Inhibition of dehydrogenase enzymes by salicylate. Nature 201: 192.

Kopp E, Ghosh S 1994. Inhibition of NF-kappa B by sodium salicylate and aspirin. Science 265: 956-959.

Kubes P, Suzuki M, Granger DN 1991. Nitric oxide: an endogenous modulator of leukocyte adhesion. Proc Natl Acad Sci USA 88: 4651-4655.

Paul-Clark MJ, Van Cao T, Moradi-Bidhendi N, Cooper D, Gilroy DW 2004. 15-epi-lipoxin A4-mediated induction of nitric oxide explains how aspirin inhibits acute inflammation. J Exp Med 200: 69-78.

Pérez-Sala D, Cernuda-Morollón E, Díaz-Cazorla M, RodríguezPascual F, Lamas S 2001. Posttranscriptional regulation of human iNOS by the NO/cGMP pathway. Am J Physiol Renal Physiol 280: F466-F473.

Perretti M, Chiang N, La M, Fierro IM, Marullo S, Getting SJ, Solito E, Serhan CN 2002. Endogenous lipid- and peptidederived anti-inflammatory pathways generated with glucocorticoid and aspirin treatment activate the lipoxin A4 receptor. Nat Med 8: 1296-1302.

Serhan CN, Oliw E 2001. Unorthodox routes to prostanoid formation: new twists in cyclooxygenase-initiated pathways. J Clin Invest 107: 1481-1489.

Smith JB, Willis AL 1971. Aspirin selectively inhibits prostaglandin production in human platelets. Nature 231: 235-237.

Tomlinson A, Appleton I, Moore AR, Gilroy DW, Willis D, Mitchell JA, Willoughby DA 1994. Cyclo-oxygenase and nitric oxide synthase isoforms in rat carrageenin- induced pleurisy. Br J Pharmacol 113: 693-698.

Topper JN, Cai J, Falb D, Gimbrone MAJ 1996. Identification of vascular endothelial genes differentially responsive to fluid mechanical stimuli: cyclooxygenase- 2 , manganese superoxide dismutase, and endothelial cell nitric oxide synthase are selectively up-regulated by steady laminar shear stress. Proc Natl Acad Sci USA 93: 10417-10422.

Vane JR 1971. Inhibition of prostaglandin synthesis as a mechanism of action for aspirin-like drugs. Nat New Biol 231: 232-235.

Vane JR, Botting R 1992. Aspirin and Other Salicylates, Chapman \& Hall, London.

Wallace JL, Zamuner SR, McKnight W, Dicay M, Mencarelli A, del Soldato P, Fiorucci S 2004. Aspirin, but not NOreleasing aspirin (NCX-4016), interacts with selective COX2 inhibitors to aggravate gastric damage and inflammation. Am J Physiol Gastrointest Liver Physiol 286: G76-G81.

Whitehouse MW, Haslam JM 1962. Ability of some antirheumatic drugs to uncouple oxidative phosphorylation. Nature 196: 1323-1324.

Williams KI, Higgs GA 1988. Eicosanoids and inflammation. $J$ Pathol 156: 101-110.

Wu KK, Sanduja R, Tsai AL, Ferhanoglu B, Loose-Mitchell DS 1991. Aspirin inhibits interleukin 1-induced prostaglandin $\mathrm{H}$ synthase expression in cultured endothelial cells. Proc Natl Acad Sci USA 88: 2384-2387. 